\title{
Fluid shear stress regulates vascular remodeling via VEGFR-3 activation, although independently of its ligand, VEGF-C, in the uterus during pregnancy
}

\author{
YANG-GYU PARK $^{1}$, JAWUN CHOI ${ }^{1}$, HYE-KANG JUNG $^{1}$, IN KYU SONG ${ }^{1}$, \\ YONGWHAN SHIN ${ }^{2}$, SANG-YOUEL PARK ${ }^{1}$ and JAE-WON SEOL ${ }^{1}$ \\ ${ }^{1}$ Biosafety Research Institute, College of Veterinary Medicine, Chonbuk National University, \\ Iksan, Jwonbuk 54596, Republic of Korea; ${ }^{2}$ Auckland International College, Auckland 0600, New Zealand
}

Received December 23, 2016; Accepted August 22, 2017

DOI: $10.3892 /$ ijmm.2017.3108

\begin{abstract}
Early pregnancy is characterized by an increase in the blood volume of the uterus for embryonic development, thereby exerting fluid shear stress (FSS) on the vascular walls. The uterus experiences vascular remodeling to accommodate the increased blood flow. The blood flow-induced FSS elevates the expression of vascular endothelial growth factors (VEGFs) and their receptors, and regulates vascular remodeling through the activation of VEGF receptor-3 (VEGFR-3). However, the mechanisms responsible for FSS-induced VEGFR-3 expression in the uterus during pregnancy are unclear. In this study, we demonstrate that vascular remodeling in the uterus during pregnancy is regulated by FSS-induced VEGFR-3 expression. We examined the association between VEGFR-3 and FSS through in vivo and in vitro experiments. In vivo experiments revealed VEGFR-3 expression in the CD31-positive region of the uterus of pregnant mice; VEGF-C (ligand for VEGFR-3) was undetected in the uterus. These results confirmed that VEGFR-3 expression in the endometrium is independent of its ligand. In vitro studies experiments revealed that FSS induced morphological changes and increased VEGFR-3 expression in human uterine microvascular endothelial cells. Thus, VEGFR-3 activation by FSS is associated with vascular remodeling to allow increased blood flow in the uterus during pregnancy.
\end{abstract}

Correspondence to: Professor Jae-Won Seol or Professor Sang-Youel Park, Biosafety Research Institute, College of Veterinary Medicine, Chonbuk National University, Iksan, Jwonbuk 54596, Republic of Korea

E-mail: jwsseol@jbnu.ac.kr

E-mail: sypark@jbnu.ac.kr

Abbreviations: FSS, fluid shear stress; HUtMECs, human uterine microvascular endothelial cells; MR, mesometrial region; AMR, anti-mesometrial region; UL, uterus lumen; VSR, venous sinus region; Em, embryo; BVD, blood vessel density

Key words: shear stress, vascular endothelial growth factor receptor-3, vascular remodeling, uterus of pregnant mice, post-implantation periods

\section{Introduction}

Fluid shear stress (FSS) is the blood flow-induced force per unit area of the blood vessel walls, which results in the vascular remodeling of blood vessels (1-4). The type of FSS may be laminar and disturbed, and the endothelial cell response varies with the type of FSS acting on the vessel wall $(5,6)$. Thus, vascular endothelial cells undergo structural alterations to adapt to the blood flow-induced FSS, thereby maintaining the homeostasis of the organ system (7).

The uterus is a special organ that undergoes periodic changes in blood vessels during the menstruation cycle and pregnancy $(8,9)$. Pregnancy is characterized by active neovascularization and vascular remodeling, with a progressive increase in the maternal blood volume $(8,10)$. There is a simultaneous increase in the blood flow to the uterus, and the endometrial vessels experience vascular remodeling to accommodate the increased blood flow $(8,11)$. Through vascular remodeling, the placenta that delivers maternal blood to the fetus is formed stably (12-14). The failure in vascular remodeling at this stage may be associated with pre-eclampsia and fetal growth retardation. Thus, vascular remodeling at the early stage of pregnancy is a necessary process for the development of the fetus (15). Recent studies have shown that endothelial cells may undergo changes in their morphologies and cytoskeletal structures to adapt to FSS (16-18). Furthermore, vascular remodeling by FSS is attributed to the expression of the vascular endothelial growth factor receptor-3 (VEGFR-3) $(18,19)$.

VEGFR-3 is a receptor of VEGF-C/D and a close homolog of VEGFR-2 $(20,21)$. It is predominantly expressed on lymphatic cells and vessels (21). Studies have indicated that the VEGF-C-mediated activation of VEGFR-3 results in the initiation of lymph angiogenesis through proliferation and migration of lymphatic cells $(22,23)$. It has been reported that VEGFR-3 is expressed in the endometrium of the uterus (24) and that VEGFR-3 expression was spatiotemporally similar to that of VEGFR-2 in the uterus during pregnancy (25).

A recent study demonstrated that angiogenesis associated with VEGFR-3 failed to occur in the absence of VEGFR-2 (26). In addition, it has been reported that VEGFR-3-mediated FSS regulates the remodeling of the arteries (18), suggestive of a 
plausible role of VEGFR-2 and VEGFR-3 in the process of vascular remodeling through the same or different stimulation. However, the mechanisms underlying vascular remodeling mediated via VEGFR-3 expression in the uterus during pregnancy are not yet fully understood.

According to previous studies $(18,26)$, we expected that VEGFR-3 expressed during pregnancy may be associated with FSS. In this study, we investigated the association between FSS and VEGFR-3, and the role of VEGFR-3 in vascular remodeling in the uterus during pregnancy. We demonstrate that VEGFR-3 is expressed in vascular endothelial cells of the endometrium in the uterus during pregrancy. We used lymphatic vessel endothelial hyaluronan receptor-1 (LYVE-1) staining to determine the expression of VEGFR-3 in lymphatic cells of the uterus. VEGFR-3 expression in the endometrium was distinct from the region expressing LYVE-1, while its expression in the myometrium coincided with the region expressing LYVE-1. Furthermore, we investigated the response of endothelial cells to FSS using an in vitro FSS model (19) and found that VEGFR-3 expression in human uterine microvascular endothelial cells (HUtMECs) is dependent on FSS.

\section{Materials and methods}

Mice. All animal experiments were performed following approval from the Animal Care Committees of Chonbuk National University, Iksan, Korea. Specific pathogen-free C57BL/6 mice were purchased from the Samtako Bio Korea (Osan, Korea). All mice were transferred and bred in pathogen-free animal facilities and fed a standard diet (PMI Laboratory Diet, Richmond, IN, USA) and provided with water ad libitum. Female mice (weighing $20 \pm 1.25 \mathrm{~g}$; aged 7 weeks; $\mathrm{n}=40$ ) and male mice (weighing $23 \pm 0.83 \mathrm{~g}$; aged 8 weeks; $\mathrm{n}=10$ ) were used in this study; female mice were mated with male mice. Copulation was indicated by the presence of vaginal plugs the following morning, and the plug day was designated as 0.5 days post coitum (dpc). The animals were divided into 4 groups as follows: the estrus non-pregnancy (ENP) group, and the 4.5, 6.5 and 8.5 dpc groups.

Histological analysis. The mice were sacrificed by the cervical dislocation method on the indicated days. Segments of the uterus containing implanted embryos were fixed in $4 \%$ paraformaldehyde (PFA) for $4 \mathrm{~h}$, followed by overnight dehydration in $20 \%$ sucrose solution. Dehydrated samples were embedded with tissue-freezing medium (Leica, Wetzlar, Germany) and the frozen blocks were cut into $20-\mu$ m-thick sections. The samples were blocked with 5\% donkey (or goat) serum in $0.03 \%$ Triton X-100 in phosphate-buffered saline (PBST) and incubated for $4 \mathrm{~h}$ at room temperature with the following primary antibodies: anti-CD31 (hamster, clone 2H8; cat. no. MAB1398Z; Millipore, Temecula, CA, USA), anti-VEGFR-3 (goat polyclonal; cat. no. AF743; R\&D Systems, Minneapolis, MN,USA), anti-VE-cadherin (mouse monoclonal; cat. no. sc-9989; Santa Cruz Biotechnology, Inc., Santa Cruz, CA, USA), anti-LYVE-1 (rabbit, cat. no. 11-034; AngioBio, Del Mar, CA, USA), and anti-VEGF-C (goat polyclonal; cat. no. sc-1881; Santa Cruz Biotechnology, Inc.). Following incubation, the samples were washed 3-5 times and incubated for $2 \mathrm{~h}$ at room temperature with the following secondary antibodies: Cy3-conjugated anti-hamster IgG (127-165-160;
Jackson ImmunoResearch Laboratories, West Grove, PA, USA), fluorescein isothiocyanate (FITC)-conjugated anti-mouse IgG (A90-216F; Bethyl Laboratories, Montgomery, TX, USA), FITC-conjugated anti-goat IgG (ab6881; Abcam, Cambridge, MA, USA) and Cy3-conjugated anti-rabbit IgG (711-615-152; Jackson ImmunoResearch Laboratories). Nuclei were stained with 4',6-diamidino-2-phenylindole (DAPI). Samples were mounted in fluorescent mounting medium (DAKO) and immunofluorescent images acquired using a Zeiss LSM510 confocal fluorescence microscope (Carl Zeiss, Oberkochen, Germany). The samples were overnight fixed in 4\% PFA for hematoxylin and eosin (H\&E) staining. The tissue was processed using standard procedures and embedded in paraffin. Tissue blocks were cut into 3- $\mu \mathrm{m}$-thick sections and subjected to H\&E staining.

Morphometric analysis. Densities and different sizes of blood vessels in the uterus of pregnant mice were analyzed using photographic analysis with ImageJ software (http://rsb. info.nih.gov/ij) and LSM Image Browser (Carl Zeiss). The CD31-positive blood vessels were measured in the venous sinus region (VSR) and presented as percentage per measured area. Different sized blood vessels were measured in the mesometrial region $(\mathrm{MR})$ of the uterus at 6.5 and $8.5 \mathrm{dpc}$.

Cell culture. HUtMECs purchased from Lonza Group, Ltd. (Basel, Switzerland) (CC-2564) were grown in endothelial cell growth medium (EGM-2 MV BulletKit, CC-3202) and used at passage 3-4 in all the experiments.

FSS model. The FSS model for in vitro experiments was used as previously described (19). The shear stress across each monolayer was approximated as the maximal wall shear stress as follows:

$\tau_{\max }=\sqrt[\alpha]{\rho \eta(2 \pi f)^{3}} \begin{aligned} & \text { where } \alpha \text { is the radius of orbital rotation } \\ & (1.25 \mathrm{~cm}), \varrho \text { and } \eta \text { are the density }(1.0 \mathrm{~g} / \mathrm{ml})\end{aligned}$ and viscosity $\left(7.5 \times 10^{-3}\right.$ dynes $\left./ \mathrm{sec} / \mathrm{cm}\right)$ of the medium, respectively, and $f$ is the frequency of rotation (rotations/sec). Using this equation, a shear stress of $10 \mathrm{dynes} / \mathrm{cm}^{2}$ was achieved at a rotating frequency of $91 \mathrm{rpm}$ (rotation/min), which was within the range of physiological shear stress $\left(0-20 \mathrm{dynes} / \mathrm{cm}^{2}\right)$. HUtMECs at the same passage stage and not subjected to shear stress were incubated in the same incubator and served as the static controls. The alignment of the HUtMECs was detected using a Nikon Eclipse TS 100 microscope with a digital camera system under a $10 \mathrm{X}$ objective.

RNA extraction and reverse transcription-quantitative (realtime) polymerase chain reaction $(R T-q P C R)$. Total RNA was extracted from the uterus using TRIzol ${ }^{\circledR}$ reagent (Invitrogen, Carlsbad,CA,USA) according to the manufacturer's instructions. The extracted RNA $(2 \mu \mathrm{g})$ was reverse transcribed into cDNA using SuperScript II Reverse Transcriptase (Invitrogen). Quantitative PCR was performed using Bio-Rad ${ }^{\mathrm{TM}}$ CFX96 Real-Time PCR detection system (Bio-Rad, Hercules, CA, USA) with the following primers: VEGFR-3 forward, 5'-CCT GAAGAAGATCGCTGTTC-3' and reverse, 5'-GAGAG CTGGTTCCTGGAGAT-3'; and glyceraldehyde 3-phosphate dehydrogenase (GAPDH; internal control) forward, 5'-TGCCT CCTGCACCACCAACT-3' and reverse, 5'-CGCCTGCTTCA CCACCTTC-3'. 
Western blot analysis. The uterus tissue and cells were homogenized in cold radioimmunoprecipitation assay (RIPA) buffer supplemented with protease inhibitor cocktail on the indicated days. Each protein was separated by sodium dodecyl sulfatepolyacrylamide gel electrophoresis (SDS-PAGE) and transferred onto nitrocellulose membranes. After blocking with 5\% skim milk, the membranes were incubated overnight with goat polyclonal anti-VEGFR-3 (dilution 1:1,000; cat. no. sc-321; Santa Cruz Biotechnology, Inc.) and mouse monoclonal anti- $\beta$-actin antibody (dilution 1:1,000; cat. no. A5441; Sigma-Aldrich, St. Louis, MO, USA) at $4^{\circ} \mathrm{C}$. Following incubation, and then probed with horseradish peroxidase (HRP)-conjugated secondary antibody [goat anti-mouse (dilution 1:5,000; cat. no. ADI-SAB-100-J) and goat anti-rabbit (dilution 1:5,000; cat. no. ADI-SAB-300); both from Enzo Life Sciences, Inc., Farmingdale, NY, USA] for $2 \mathrm{~h}$ at room temperature and the signal developed with the enhanced chemiluminescence HRP substrate (Millipore) was detected using the Fusion FX7 acquisition system (Vilbert Lourmat, Eberhardzell, Germany).

Immunocytochemistry. The HUtMECs were cultured on glass slides, fixed with cold acetone, and blocked with 5\% donkey serum in Tris-buffered saline, $0.1 \%$ Tween-20 (TBST). The cells were incubated with goat polyclonal anti-VEGFR-3 (goat polyclonal; cat. no. AF743; R\&D Systems) and mouse monoclonal anti-VE-cadherin (mouse monoclonal; cat. no. sc-9989; Santa Cruz Biotechnology, Inc.) at $4^{\circ} \mathrm{C}$, followed by treatment with FITC-conjugated anti-goat IgG (cat. no. ab6881; Abcam) and Cy3-conjugated anti-mouse IgG (cat. no. 715-165-150; Jackson ImmunoResearch Laboratories). Nuclei were stained with DAPI (cat. no. BML-AP402; Enzo Life Sciences, Inc.). Samples were mounted in a fluorescent mounting medium and immunofluorescent images were acquired using a Zeiss LSM510 confocal fluorescence microscope.

Statistical analysis. Values are presented as the means \pm standard deviation (SD). Statistical significance between groups was determined using an unpaired Student's t-test or one-way analysis of variance (ANOVA) followed by the StudentNewman-Keuls test. Statistical significance was set at $\mathrm{p}<0.05$ or $\mathrm{p}<0.01$.

\section{Results}

The endometrium undergoes gradual vascular remodeling during early pregnancy. We obtained a fertilized uterine sample to observe morphological changes in the uterus during early pregnancy (Fig. 1). According to the embryo morphology, we divided the uterus into the MR and anti-mesometrial region (AMR); MR was further subdivided into the uterus lumen (UL) region and VSR. Immunofluorescence staining using CD31 antibody, an endothelial cell marker, was performed to observe changes in blood vessels in the uterus of pregnant mice. As shown in Fig. 1A, the changes in blood vessels within the uterus of pregnant mice were observed. A higher magnification revealed significant changes in the vascular diameter and size of the VSR. The blood vessel density (BVD) in the VSR gradually increased by 14.7 and $27.3 \%$ at 6.5 and $8.5 \mathrm{dpc}$, respectively, as compared with that at $4.5 \mathrm{dpc}$ (Fig. 1B). Within the MR, the number of blood vessels with a diameter $>300 \mu \mathrm{m}$ increased, while those with a diameter $<300 \mu \mathrm{m}$ decreased at $8.5 \mathrm{dpc}$ compared to $6.5 \mathrm{dpc}$ (Fig. 1C). In addition, significant morphological changes were observed in the uterus during early pregnancy (Fig. 1D). There was a gradual increase in the proportion of embryos in the uterus, and the vascular lumen of the MR became enlarged and elonged. In particular, changes in the VSR within the uterus were easily noticeable (Fig. 1E). These results demonstrate that blood vessels in the endometrium of uterus undergo neovascularization and vascular remodeling during pregnancy.

Expression of VEGFR-3 in the endometrium gradually increases during pregnancy. It has been reported that vascular remodeling within the uterus is controlled with progesterone-mediated VEGF-A/VEGFR-2 signaling (25). In addition, it has been shown that angiogenesis associated with VEGF-3 fails to occur in the absence of VEGFR-2 (26). Therefore, we hypothesized that VEGFR-3 plays a role in the vascular remodeling of the uterus during pregnancy. Our results revealed an increase in the expression of VEGFR-3 in the uterus during the post-implantation periods (Fig. 2C). In comparison to the level observed $4.5 \mathrm{dpc}$, VEGFR-3 expression exhibited an increase of approximately $60 \%$ at $8.5 \mathrm{dpc}$ (Fig. 2D). Furthermore, VEGFR-3 was predominantly expressed in the myometrium and MR of the endometrium (Fig. 2A). In particular, VEGFR-3 exhibited a strong expression in the VSR and myometrium. VEGFR-3 expression in the endometrium coincided with a CD31-positive region (Fig. 2B). These results suggest that VEGFR-3 expression in the endometrium may be involved in vascular remodeling during post-implantation periods.

VEGFR-3 expression in the endometrium is not associated with lymphatic vasculature and is independent of VEGF-C expression. VEGFR-3 is known to be strongly expressed in lymphatic endothelial cells. Therefore, we evaluated the expression of VEGFR-3 in lymphatic cells by immunofluorescence staining of VEGFR-3 using LYVE-1 (a lymphatic endothelial cell marker) (Fig. 3A). We confirmed VEGFR-3 expression in the region of the myometrium which stained positive for LYVE-1. On the contrary, VEGFR-3 expression in the endometrium failed to coincide with the region exhibiting LYVE-1 expression (Fig. 3C). Furthermore, VEGF-C and LYVE-1 were co-stained to evaluate the expression of VEGF-C, a ligand for VEGFR-3, in the lymphatic endothelial cells of the uterus (Fig. 3B and D). The staining results confirmed that VEGF-C exhibited no detectable expression in the endometrium. In addition, the protein expression of VEGF-C was hardly detected (Fig. 3E). We confirmed the same results as those of a previous study (25). Taken together, these results demonstrate that VEGFR-3 is expressed in vascular endothelial cells undergoing vascular remodeling during early pregnancy and is independent of the expression of its ligand, VEGF-C.

FSS induces VEGFR-3 expression in HUtMECs. To determine whether VEGFR-3 expression in vascular endothelial cells of the endometrium actually responds to FSS, HUtMECs were used to observe the effects of FSS on vascular endothelial cells. The FSS experiments were performed using the method described in a previous study (19). Our results revealed that 
A
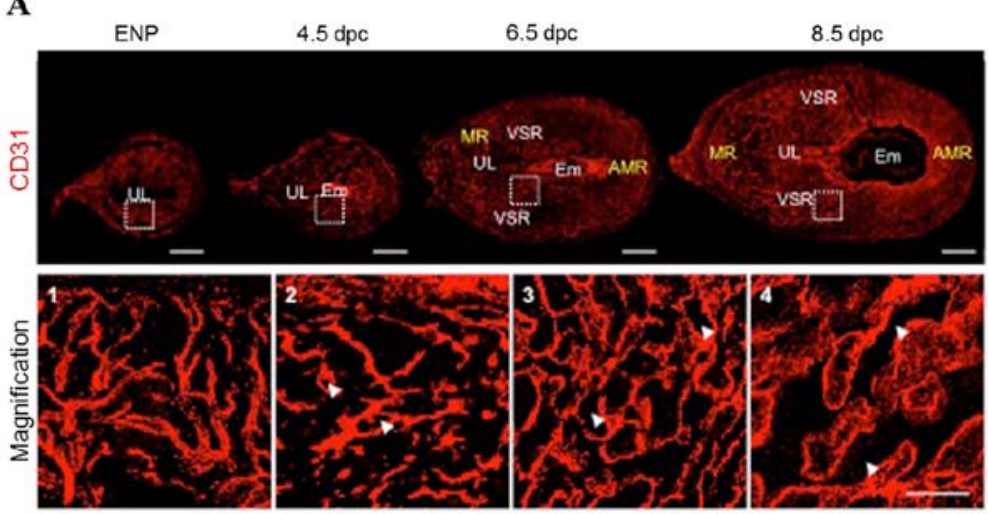

D
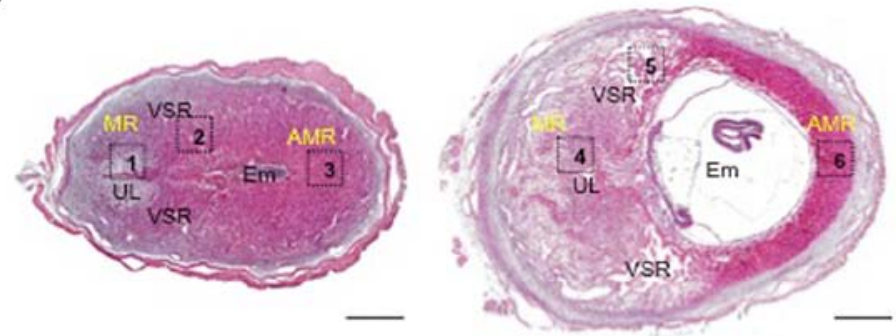

B
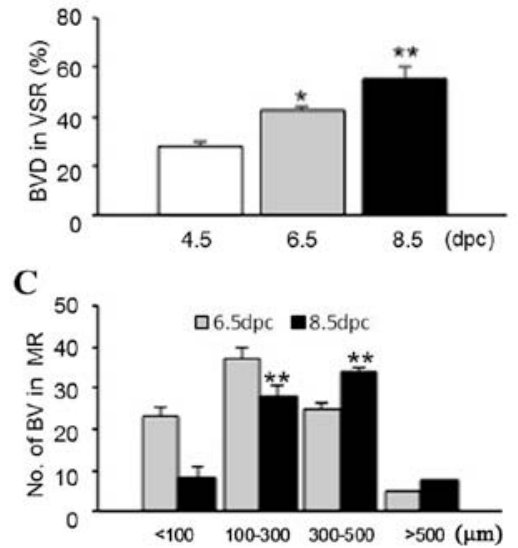

E

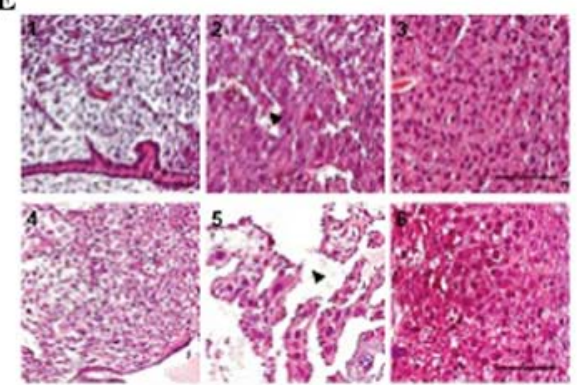

Figure 1. Changes (vascular remodeling) occurring in the endometrium during pregnancy. (A) Images showing CD31+ BVs in the uterus at ENP, 4.5, 6.5 and 8.5 days post coitum (dpc). Scale bars, $500 \mu \mathrm{m}$. Each numbered magnification image (square-dotted line) shows endometrial CD31 ${ }^{+}$BVs in the VSR. Scale bars, $100 \mu \mathrm{m}$. (B) Comparisons of CD31+ BV densities (BVD, \%) in the VSR at 4.5, 6.5 and $8.5 \mathrm{dpc}$. Each group, $\mathrm{n}=5-6 .{ }^{*} \mathrm{p}<0.05 \mathrm{vs} .4 .5 \mathrm{dpc} ;{ }^{* *} \mathrm{p}<0.01 \mathrm{vs} .4 .5 \mathrm{dpc}$ by unpaired t-test. (C) Comparisons of numbers of different sized BVs in the MR at 6.5 and $8.5 \mathrm{dpc}$. Each group, n=5-6. " $\mathrm{p}<0.05 \mathrm{vs} .6 .5 \mathrm{dpc}$; ${ }^{* *} \mathrm{p}<0.01 \mathrm{vs} .6 .5 \mathrm{dpc}$ by unpaired t-test. (D) Cross-sectioned uterus from 6.5 to $8.5 \mathrm{dpc}$ stained with hematoxylin and eosin. Scale bars, $500 \mu \mathrm{m}$. (E) Magnified images showing enlarged and elongated vascular lumen (arrowheads) in the uterus at 6.5 to $8.5 \mathrm{dpc}$. Scale bars, $100 \mu \mathrm{m}$. MR, mesometrial region; AMR, anti-mesometrial region; UL, uterus lumen; VSR, venous sinus region; Em, embryo; BVs, blood vessels.

A

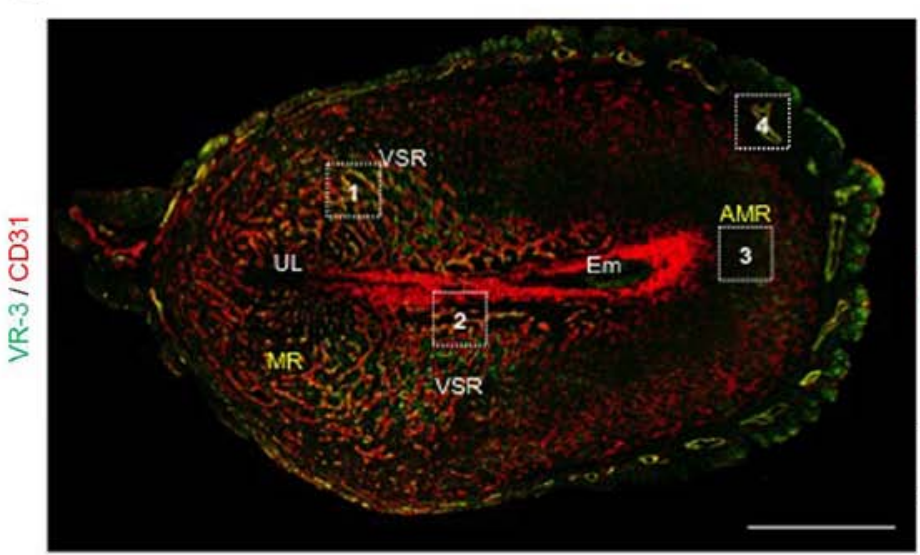

B

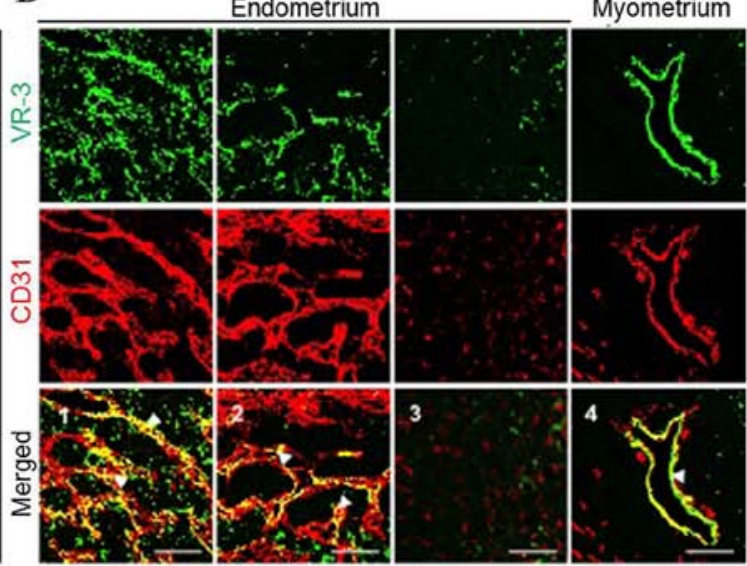

C

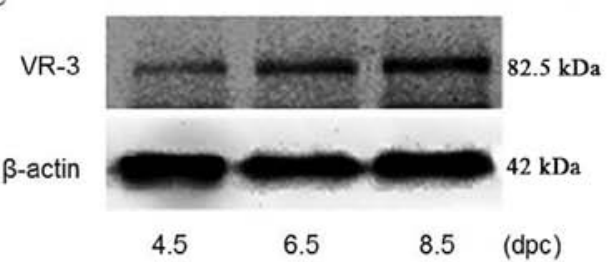

D

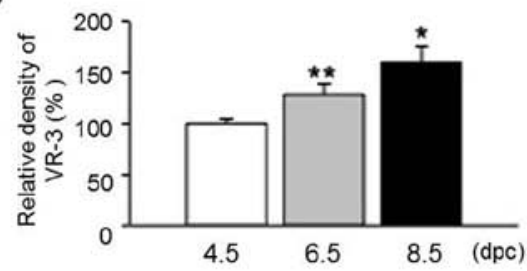

Figure 2. Vascular endothelial growth factor receptor-3 (VEGFR-3) expression increases during pregnancy in the uterus. (A) Image showing CD31+ BVs and VEGFR- $3^{+}$cells in the uterus at 6.5 days post coitum (dpc). Scale bars, $500 \mu \mathrm{m}$. (B) Magnified images showing VEGFR-3 expressed CD31 ${ }^{+}$BVs (arrowheads) in the endometrium and myometrium of uterus at $6.5 \mathrm{dpc}$. Scale bars, $50 \mu \mathrm{m}$ (C) Comparison of VEGFR-3 protein levels in the uterus at 4.5 to $8.5 \mathrm{dpc}$. Levels of VEGFR-3 were normalized to those of $\beta$-actin. (D) The VEGFR-3 protein levels in the uterus at 4.5 to $8.5 \mathrm{dpc}$ as shown in (C). Each group, $n=5-6 .{ }^{*} \mathrm{p}<0.05 \mathrm{vs}$. $4.5 \mathrm{dpc}$; ${ }^{* *}<<0.01$ vs. 4.5 dpc by unpaired t-test. VR-3, VEGFR-3; MR, mesometrial region; AMR, anti-mesometrial region; UL, uterus lumen; VSR, venous sinus region; Em, embryo. 
A

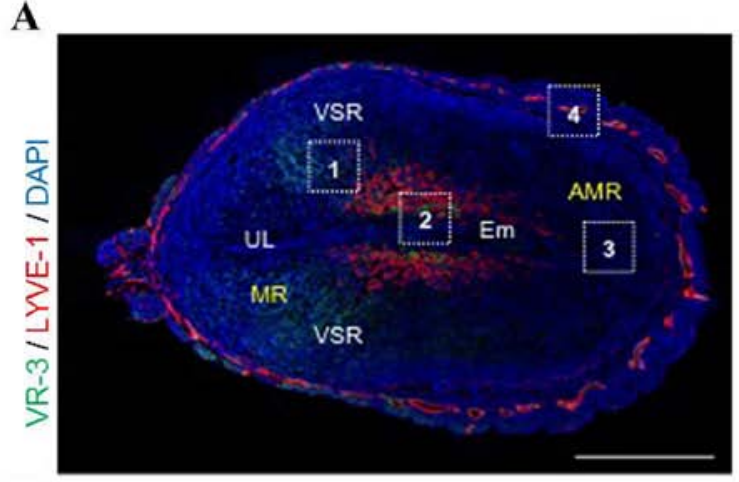

B

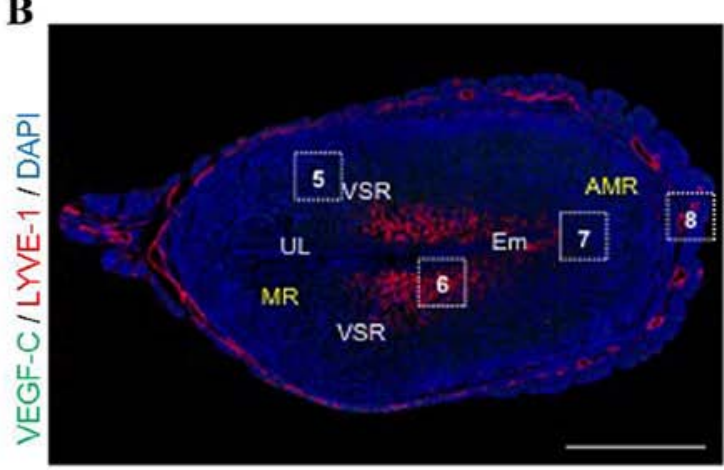

C

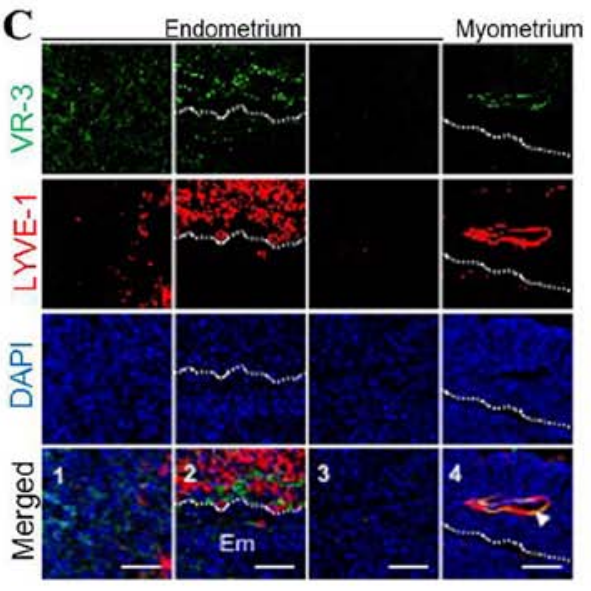

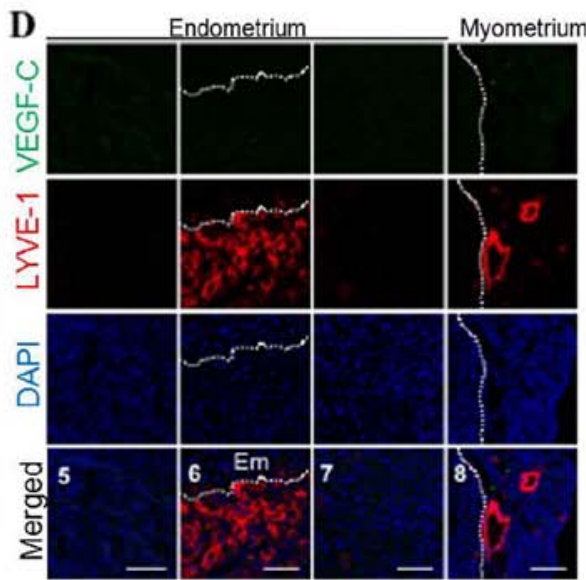

E

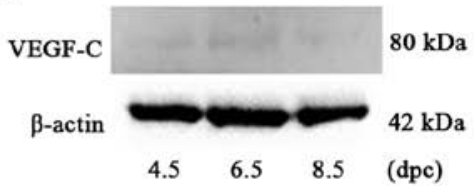

Figure 3. Vascular endothelial growth factor receptor-3 (VEGFR-3) expression in the endometrium does not coincide with that of lymphatic vessel endothelial hyaluronan receptor-1 (LYVE-1) and is independent of VEGF-C. (A) Image showing LYVE- $1^{+}$regions and VEGFR-3 ${ }^{+}$regions in the uterus at 6.5 days post coitum (dpc). Scale bars, $500 \mu \mathrm{m}$. (B) Image showing LYVE-1+ regions and VEGF-C ${ }^{+}$regions in the uterus at $6.5 \mathrm{dpc}$. Scale bars, $500 \mu \mathrm{m}$. (C) Magnified images showing VEGFR- $3^{+}$regions and LYVE-1 $1^{+}$regions in the endometrium and myometrium of uterus at $6.5 \mathrm{dpc}$. Scale bars, $50 \mu \mathrm{m}$. (D) Magnified images showing VEGF-C ${ }^{+}$regions and LYVE-1 ${ }^{+}$regions in the endometrium and myometrium of uterus at $6.5 \mathrm{dpc}$. Scale bars, $50 \mu \mathrm{m}$. (E) Comparison of VEGF-C protein levels in the uterus of 4.5-8.5 dpc. Levels of VEGF-C were normalized to those of $\beta$-actin. MR, mesometrial region; AMR, anti-mesometrial region; UL, uterus lumen; VSR, venous sinus region; Em, embryo.

VEGFR-3 expression in the vascular endothelial cells was regulated by FSS. Changes in the morphology of the HUtMECs were observed at varying FSS strengths (Fig. 4A); an increase in FSS resulted in the alignment of cells in the direction of the flow. Cells subjected to direct FSS exhibited a strong expression of VEGFR-3 around the nucleus and exhibited morphological changes (Fig. 4F). Furthermore, FSS increased the mRNA expression of VEGFR-3, as well as its protein expression (Fig. 4B and D). Based on the static controls, in the cells exposed to FSS at 10 and 20 dyne $/ \mathrm{cm}^{2}$, mRNA expression increased by approximately 85.4 and $100.5 \%$, respectively, and the protein expression increased by approximately 12.9 and 85.8\%, respectively (Fig. 4C and E). In addition, VEGF-C was not found to be expressed in the HUtMECs (Fig. 4G). These results confirm that the vascular remodeling induced by FSS in vascular endothelial cells is dependent on VEGFR-3 expression, but not on that of its ligand, VEGF-C.

\section{Discussion}

Vascular remodeling is a process that occurs to maintain the homeostasis of blood vessels by adapting to changes in blood flow and is an essential process for the survival of vascular endothelial cells on blood vessels (7). Thus, neovascularization and vascular remodeling are frequently observed in developing tissues or organs $(8,9)$. The uterus is a special organ that experiences periodic vascular remodeling due to menstruation $(8,10,11)$. The uterus exhibits marked neovascularization and vascular remodeling during early pregnancy, which promotes the stable development of the embryo $(13,14)$. The failure of this process may be associated with pre-eclampsia, miscarriage and fetal growth restriction $(25,27)$. Therefore, remodeling of the blood vessels occurring in the early stages of pregnancy is a very important process for embryonic development. During pregnancy, the maternal body experiences increased levels of hormones, such as estrogen and progesterone and increased body temperature and blood flow $(8,25)$. Due to changes in blood flow, the blood vessel wall is subject to stimulations, such as increased blood pressure and FSS, through which the blood vessel is remodeled to adapt to the changed blood flow $(3,4,17)$.

A previous study demonstrated that progesterone governs vascular remodeling via VEGF-A/VEGFR-2 signaling during early pregnancy (25). The expression of VEGFR-3 in the uterus is spatiotemporally similar to that of VEGF-2. However, the association between VEGFR-3 and vascular remodeling during early pregnancy is not yet fully understood. Recently, FSS has been shown to activate VEGFR-3 and regulate vascular remodeling in a VEGFR-3-dependent manner (18). In addition, it has been demonstrated angiogenesis associated with VEGFR-3 failed to occur in the absence of VEGFR-2 (26). All these data 
A

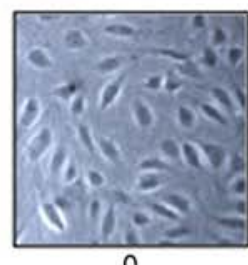

0

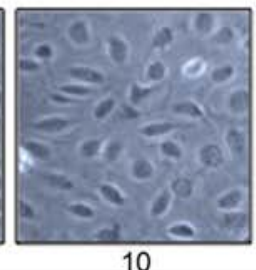

dyne $/ \mathrm{cm}^{2}$

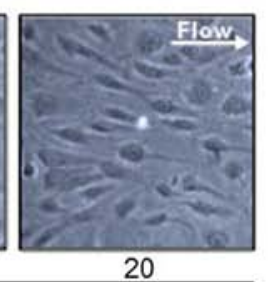

B

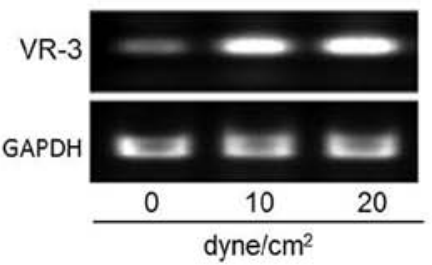

C

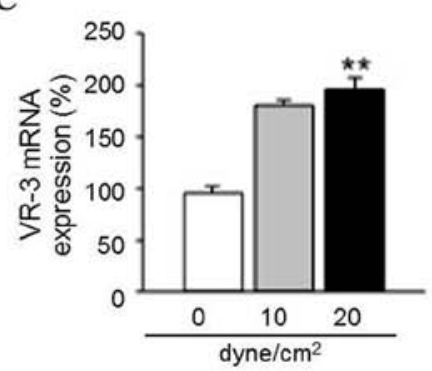

D

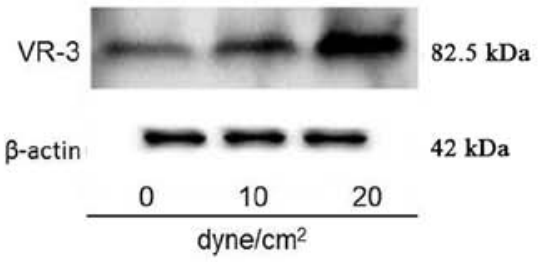

E

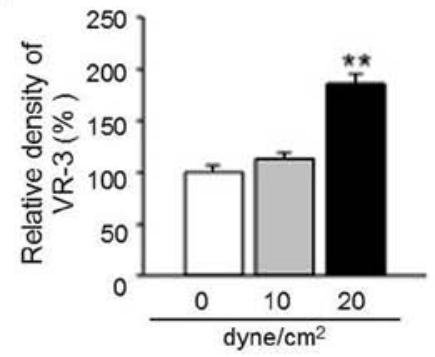

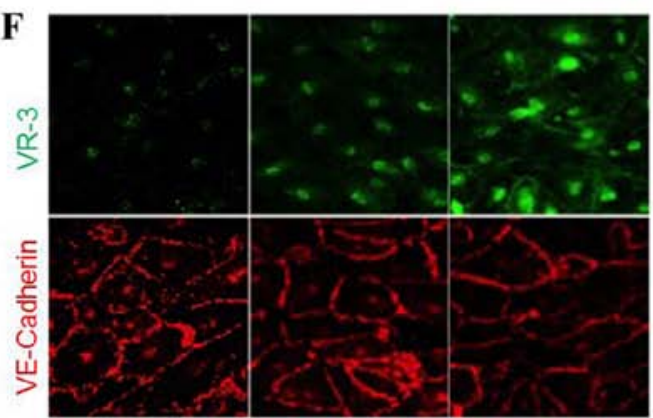

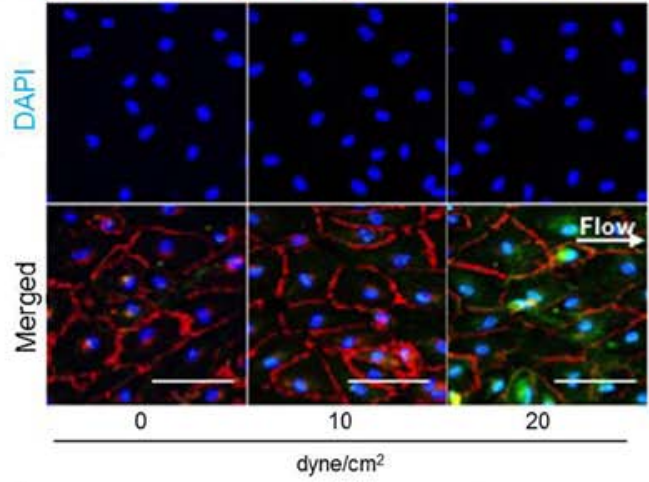

G

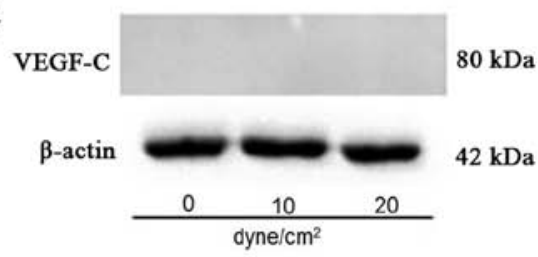

Figure 4. Fluid shear stress regulates vascular endothelial growth factor receptor-3 (VEGFR-3) expression in human uterine microvascular endothelial cells (HUtMECs). (A) Morphology of HUtMECs following $24 \mathrm{~h}$ of exposure to static $\left(0\right.$ dyne $\left./ \mathrm{cm}^{2}\right)$ and fluid shear stress (FSS) $\left(10-20 \mathrm{dyne} / \mathrm{cm}^{2}\right) \mathrm{condition}$ (x100 magnification). (B) Comparison of VEGFR-3 mRNA expression in HUtMECs after $24 \mathrm{~h}$ exposure to static $\left(0 \mathrm{dyne} / \mathrm{cm}^{2}\right)$ and FSS $\left(10-20 \mathrm{dyne} / \mathrm{cm}^{2}\right)$ conditions. Levels of VEGFR-3 were normalized to GAPDH. (C) The vascular endothelial growth factor receptor-3 (VEGFR-3) mRNA levels in human uterine microvascular endothelial cells (HUtMECs) as described in (B). ${ }^{* *} \mathrm{p}<0.01$ vs. controls $\left(0\right.$ dyne/ $\left.\mathrm{cm}^{2}\right)$ by unpaired t-test. (D) Comparison of VEGFR-3 protein expression in HUtMECs after $24 \mathrm{~h}$ exposure to static $\left(0\right.$ dyne $\left./ \mathrm{cm}^{2}\right)$ and fluid shear stress (FSS) $\left(10-20 \mathrm{dyne} / \mathrm{cm}^{2}\right)$ conditions. Levels of VEGFR-3 were normalized to $\beta$-actin. (E) The VEGFR-3 protein levels in HUtMECs as described in (D). ${ }^{* *} \mathrm{p}<0.01$ vs. controls $\left(0\right.$ dyne $\left./ \mathrm{cm}^{2}\right)$ by unpaired t-test. (F) Representative immunocytochemistry of HUtMECs after $24 \mathrm{~h}$ exposure to static $\left(0\right.$ dyne $\left./ \mathrm{cm}^{2}\right)$ and FSS $\left(10-20\right.$ dyne $\left./ \mathrm{cm}^{2}\right)$ conditions to determine VEGFR-3 and VE-Cadherin protein levels. Scale bars, $100 \mu \mathrm{m}$. (G) Comparison of VEGF-C protein expression in HUtMECs after $24 \mathrm{~h}$ exposure to static $\left(0\right.$ dyne/ $\left./ \mathrm{cm}^{2}\right)$ and FSS $(10-20$ dyne/ $\left.\mathrm{cm}^{2}\right)$ conditions. Levels of VEGF-C were normalized to those of $\beta$-actin.

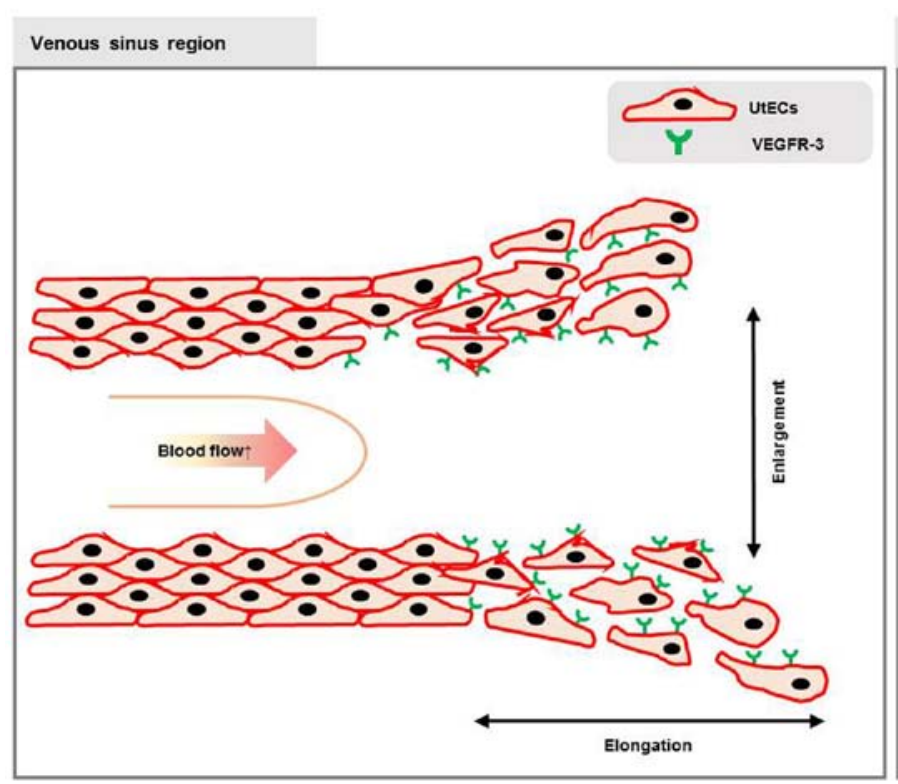

Uterine endothelial cells

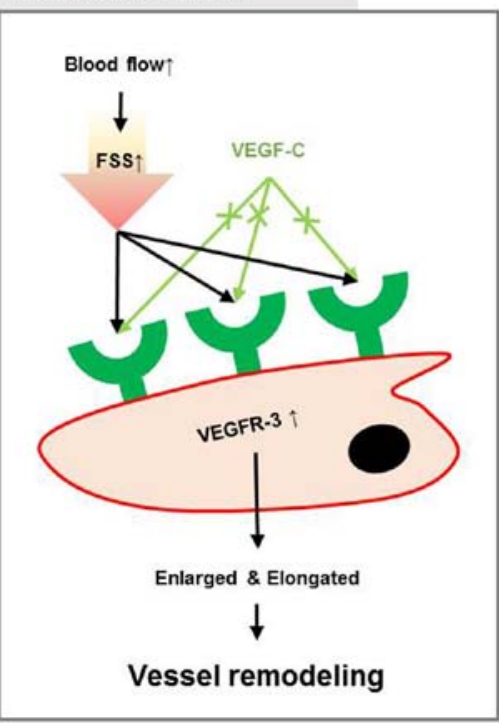

Figure 5. Schematic diagram of uterus vascular remodeling (venous sinus region in MR) by fluid shear stress via ligand independent vascular endothelial growth factor receptor-3 (VEGFR-3) activation. 
suggest that VEGFR-3 may play an important role in vascular remodeling within the uterus during pregnancy and that FSS may regulate its expression. Thus, we hyopthesized that VEGFR3-related signaling may be involved in vascular remodeling.

In this study, we examined the association between FSS and VEGFR-3 expression in uterus of pregnant mice (Fig. 5). Prior to demonstrating our prediction, we observed vascular remodeling within the uterus and identified areas where vascular remodeling was actively occurring (Fig. 1). As expected, VSR characterized by active vascular remodeling exhibited a high expression level of VEGFR-3 (Fig. 2). We confirmed VEGFR-3 expression in the uterus of pregnant mice and observed an increase in its expression during post-implantation periods (Fig. 2). Both blood and lymph vessels in the uterus were positive for VEGFR-3 expression. VEGFR-3 expression in the myometrium coincided with the region expressing LYVE-1; however, in the endometrium, VEGFR expression was confirmed in the CD31-positive region (Fig. 3A and C). VEGF-C and LYVE-1 were co-stained to evaluate the expression of VEGF-C in lymphatic endothelial cells of the uterus (Fig. 3B and D). As a result, it was confirmed that VEGF-C was barely observed in the uterus. Our results revealed that VEGFR-3 expression in vascular endothelial cells of the endometrium was independent of VEGF-C expression. We confirmed the effect of FSS on the expression of VEGFR-3 in vascular endothelial cells using a previously described shear stress model (19). Changes in FSS increased the expression of VEGFR-3 and induced morphological changes (cell elongation) in HUtMECs.

Taken together, both progesterone-mediated VEGF-A/ VEGFR-2 signaling and FSS-induced VEGFR-3 expression are thought to be involved in the regulation of vascular remodeling in the uterus during pregnancy. However, the correlation between VEGFR-3 and vascular remodeling was not clarified clearly. Further studies are required to focus on the relationship between VEGFR-3 and vascular remodeling. Nevertheless, our results indicated that VEGFR-3 expression is observed in vascular endothelial cells of the uterus and that blood flowinduced FSS contribute to the process of vascular remodeling through the regulation of VEGFR-3 expression. Thus, changes in blood flow can affect the intrauterine environment and regulation of VEGFR-3 expression to induce vascular remodeling may prevent pre-eclampsia, miscarriage and fetal growth restriction.

\section{Acknowledgements}

This study was supported by the National Research Foundation of Korea funded by the Ministry of Science, ICT and Future Planning (2015R1A1A1A05001546, to J.-W.S.) and by the Korean Institute of Planning and Evaluation for Technology in Food, Agriculture, Forestry and Fisheries (IPET) through Agriculture, Food and Rural Affairs Research Center Support Program, funded by Ministry of Agriculture, Food and Rural Affairs (MAFRA; 716002-7).

\section{References}

1. Baeyens N, Bandyopadhyay C, Coon BG, Yun S and Schwartz MA: Endothelial fluid shear stress sensing in vascular health and disease. J Clin Invest 126: 821-828, 2016.

2. Galie PA, Nguyen DH, Choi CK, Cohen DM, Janmey PA and Chen CS: Fluid shear stress threshold regulates angiogenic sprouting. Proc Natl Acad Sci USA 111: 7968-7973, 2014.
3. Ghaffari S, Leask RL and Jones EA: Flow dynamics control the location of sprouting and direct elongation during developmental angiogenesis. Development 142: 4151-4157, 2015.

4. Davies PF: Hemodynamic shear stress and the endothelium in cardiovascular pathophysiology. Nat Clin Pract Cardiovase Med 6: 16-26, 2009.

5. Chiu JJ and Chien S: Effects of disturbed flow on vascular endothelium: Pathophysiological basis and clinical perspectives. Physiol Rev 91: 327-387, 2011.

6. Heo KS, Fujiwara K and Abe J: Shear stress and atherosclerosis. Mol Cells 37: 435-440, 2014.

7. Zhang J and Friedman MH: Adaptive response of vascular endothelial cells to an acute increase in shear stress frequency. Am J Physiol Heart Circ Physiol 305: H894-H902, 2013.

8. Osol G and Moore LG: Maternal uterine vascular remodeling during pregnancy. Microcirculation 21: 38-47, 2014.

9. Mandala M and Osol G: Physiological remodelling of the maternal uterine circulation during pregnancy. Basic Clin Pharmacol Toxicol 110: 12-18, 2012.

10. Nakamura H, Hosono T, Minato K, Hamasaki T, Kumasawa K and Kimura T: Importance of optimal local uterine blood flow for implantation. J Obstet Gynaecol Res 40: 1668-1673, 2014.

11. Wang L, Qiao J, Li R, Zhen X and Liu Z: Role of endometrial blood flow assessment with color Doppler energy in predicting pregnancy outcome of IVF-ET cycles. Reprod Biol Endocrinol 8: 122, 2010.

12. Rai A and Cross JC: Development of the hemochorial maternal vascular spaces in the placenta through endothelial and vasculogenic mimicry. Dev Biol 387: 131-141, 2014.

13. Sipos PI, Rens W, Schlecht H, Fan X, Wareing M, Hayward C, Hubel CA, Bourque S, Baker PN, Davidge ST, et al: Uterine vasculature remodeling in human pregnancy involves functional macrochimerism by endothelial colony forming cells of fetal origin. Stem Cells 31: 1363-1370, 2013.

14. Soares MJ, Chakraborty D, Kubota K, Renaud SJ and Rumi MA: Adaptive mechanisms controlling uterine spiral artery remodeling during the establishment of pregnancy. Int J Dev Biol 58: 247-259, 2014.

15. Roberts JM and Escudero C: The placenta in preeclampsia. Pregnancy Hypertens 2: 72-83, 2012.

16. Conway DE, Breckenridge MT, Hinde E, Gratton E, Chen CS and Schwartz MA: Fluid shear stress on endothelial cells modulates mechanical tension across VE-cadherin and PECAM-1. Curr Biol 23: 1024-1030, 2013.

17. Steward R Jr, Tambe D, Hardin CC, Krishnan R and Fredberg JJ: Fluid shear, intercellular stress, and endothelial cell alignment. Am J Physiol Cell Physiol 308: C657-C664, 2015.

18. Baeyens N, Nicoli S, Coon BG, Ross TD, Van den Dries K, Han J, Lauridsen HM, Mejean CO, Eichmann A, Thomas JL, et al: Vascular remodeling is governed by a VEGFR3-dependent fluid shear stress set point. eLife 4: e04645., 2015.

19. dela Paz NG, Walshe TE, Leach LL, Saint-Geniez M and D'Amore PA: Role of shear-stress-induced VEGF expression in endothelial cell survival. J Cell Sci 125: 831-843, 2012.

20. Shibuya M: Vascular endothelial growth factor (VEGF) and its receptor (VEGFR) signaling in angiogenesis: A crucial target for anti- and pro-angiogenic therapies. Genes Cancer 2: 1097-1105, 2011.

21. Davydova N, Harris NC, Roufail S, Paquet-Fifield S, Ishaq M, Streltsov VA, Williams SP, Karnezis T, Stacker SA and Achen MG: Differential receptor binding and regulatory mechanisms for the lymphangiogenic growth factors VEGF-C and VEGF-D. J Biol Chem 291: 27265-27278, 2016.

22. Rutkowski JM, Ihm JE, Lee ST, Kilarski WW, Greenwood VI, Pasquier MC, Quazzola A, Trono D, Hubbell JA and Swartz MA: VEGFR-3 neutralization inhibits ovarian lymphangiogenesis, follicle maturation, and murine pregnancy. Am J Pathol 183: 1596-1607, 2013.

23. Coso S, Bovay E and Petrova TV: Pressing the right buttons: Signaling in lymphangiogenesis. Blood 123: 2614-2624, 2014.

24. Wang J, Taylor A, Showeil R, Trivedi P, Horimoto Y, Bagwan I, Ewington L, Lam EW and El-Bahrawy MA: Expression profiling and significance of VEGF-A, VEGFR2, VEGFR3 and related proteins in endometrial carcinoma. Cytokine 68: 94-100, 2014.

25. Kim M, Park HJ, Seol JW, Jang JY, Cho YS, Kim KR, Choi Y, Lydon JP, Demayo FJ, Shibuya M, et al: VEGF-A regulated by progesterone governs uterine angiogenesis and vascular remodelling during pregnancy. EMBO Mol Med 5: 1415-1430, 2013.

26. Zarkada G, Heinolainen K, Makinen T, Kubota Y and Alitalo K: VEGFR3 does not sustain retinal angiogenesis without VEGFR2. Proc Natl Acad Sci USA 112: 761-766, 2015.

27. Saito $S$ and Nakashima A: A review of the mechanism for poor placentation in early-onset preeclampsia: The role of autophagy in trophoblast invasion and vascular remodeling. J Reprod Immunol 101-102: 80-88, 2014. 